\title{
Specific symptoms in adolescence predict psychosis in the Northern Finland Birth Cohort 1986
}

Johanna Palomäki, Martta Kerkelä, Marjo-Riitta Järvelin, Tanja Nordström, Juha Veijola

Department of Psychiatry, Research Unit of Clinical Neuroscience, University of Oulu, Finland johanna.palomaki@student.oulu.fi

Background: A number of psychological symptoms have been found to predict psychosis. Many studies have found no specificity to separate symptoms predicting non-psychotic psychiatric disorders from those predicting psychotic disorders. We were able to conduct prospective study comparing adolescent symptoms predicting non-psychotic psychiatric disorders and psychotic psychiatric disorders. Our plan was to find out what kind of symptoms in adolescence predict first episode psychosis in general population.

Methods: Members of the Northern Finland Birth Cohort 1986 were asked to fill in PROD-screen questionnaire at age 15-16 years. PROD-screen includes 21 items both measuring positive prodromal symptoms, negative prodromal symptoms and general symptoms. We were able to follow 5,368 participants using Finnish Hospital Discharge Register detecting new hospital treated mental disorder till 30 years.
Table 1. PROD-screen symptoms at age 16 predicting onset of psychosis vs non-psychotic disorder until age 30 in the Northern Finland 1986 Birth Cohort $(\mathrm{N}=5368)$

\begin{tabular}{|c|c|c|c|c|c|}
\hline \multirow[b]{4}{*}{$\begin{array}{l}\text { Experience: Yes } \\
\text { Total \% }\end{array}$} & \multicolumn{5}{|c|}{ Psychiatric disorder } \\
\hline & \multirow{3}{*}{$\begin{array}{l}\text { Psychosis } \\
2002-16 \\
\mathrm{~N}=83 \\
\\
100 \%\end{array}$} & \multirow{3}{*}{ 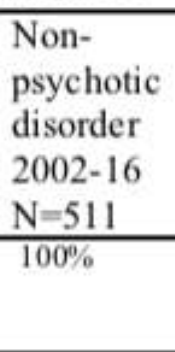 } & \multirow{3}{*}{$\begin{array}{l}\begin{array}{l}\text { No } \\
\text { disorder } \\
\mathrm{N}=4745\end{array} \\
100 \%\end{array}$} & \multicolumn{2}{|c|}{$\begin{array}{l}\text { Psychosis 2002-16 } \\
\text { vs Non-psychotic } \\
\text { disorder 2002-16 }\end{array}$} \\
\hline & & & & & \\
\hline & & & & $\begin{array}{l}\mathrm{Adj}^{\mathrm{O}} \mathrm{OR} \\
(95 \% \mathrm{Cl})\end{array}$ & $\begin{array}{l}\text { Adj. } \\
\text { p- } \\
\text { value }\end{array}$ \\
\hline \multicolumn{6}{|l|}{ Positive symptoms: } \\
\hline $\begin{array}{l}\text { "C8. Difficulties in understanding written text } \\
\text { or speech heard } 882(16.5 \%)\end{array}$ & $26.5 \%$ & $18.8 \%$ & $16.1 \%$ & $1.81(1.05-3.15)$ & 0.0340 \\
\hline $\begin{array}{l}{ }^{2} \mathrm{C} 10 \text {. Difficulty in controlling one's speech, } \\
\text { behaviour or facial expression while } \\
\text { communicating } 425(7.9 \%)\end{array}$ & $18.1 \%$ & $10.3 \%$ & $7.5 \%$ & $2.42(1.25-4.69)$ & 0.0090 \\
\hline $\begin{array}{l}\text { A20. Feelings, thoughts or behaviors that } \\
\text { could be considered weird or peculiar } 764 \\
(14.3 \%)\end{array}$ & $27.7 \%$ & $19.5 \%$ & $13.5 \%$ & $1.78(1.03-3.08)$ & 0.0390 \\
\hline
\end{tabular}

"C11. Difficulty or uncertainty in making contact with other people $514(9.6 \%)$

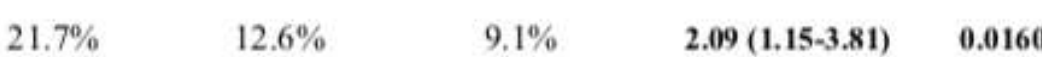

C12. Lack of initiative or difficulty in completing tasks $99(1.8 \%)$

$6.0 \%$

$1.7 \%$

$1.85(1.05-3.27) \quad 0.0350$

C18. Difficulties in carrying out ordinary routine activities (at least one week) 668 $(12.5 \%)$

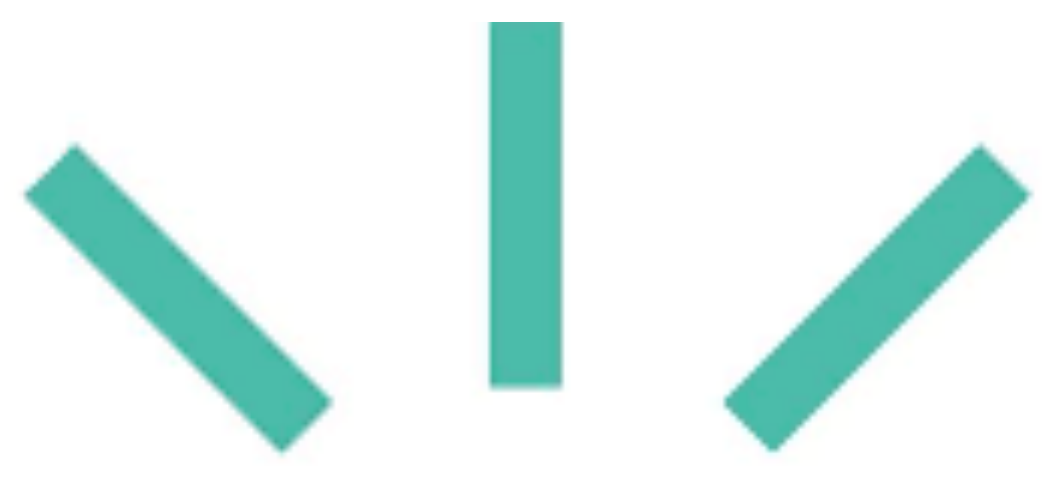

\section{NORTHERN FINLAND BIRTH COHORTS} UNIVERSITY
Results: When comparing separate symptoms in those having psychiatric hospital treatments, we found three positive symptoms and three negative symptoms predicting specifically psychotic disorders (Table 1).

Conclusions: In this large prospective population sample we found symptoms in adolescence associated specifically with development of first episode psychosis. 